\title{
Hydrogen sulfide and SIRT3 gene, the strong preventive and therapeutic agent in aging and age related diseases
}

\author{
Md. Shamim Hossain 1, *, Md. Ashrafuzzaman Sapon ${ }^{1,2}$, Naim Hassan ${ }^{1}$ \\ ${ }^{1}$ Department of Biotechnology and Genetic Engineering, Faculty of Applied Science and Technology, Islamic University, Kushtia-7003, \\ Bangladesh \\ ${ }^{2}$ Bioscience Division, Graduate school of Science \& Technology, Shizuoka University, Shizuoka, Japan
}

Email address:

shamim_btge@yahoo.com (M. S. Hossain)

\section{To cite this article:}

Md. Shamim Hossain, Md. Ashrafuzzaman Sapon, Naim Hassan. Hydrogen Sulfide and SIRT3 Gene, the Strong Preventive and Therapeutic Agent in Aging and Age Related Diseases. American Journal of BioScience. Vol. 2, No. 6, 2014, pp. 222-232.

doi: 10.11648/j.ajbio.20140206.16

\begin{abstract}
There are seven SIRT isoforms in mammals, with different biological activities including gene regulation, metabolism and apoptosis. Despite recent controversy about sirtuins function in some organisms, among them SIRT3 is the only sirtuin whose increased expression has been shown to correlate with an extended life span in humans. SIRT3 is a member of the sirtuin family of $\mathrm{NAD}^{+}$dependent deacetylases, which is localized to the mitochondria and is enriched in kidney, brown adipose tissue, heart, and other metabolically active tissues. It is an endogenous negative regulator of cardiac hypertrophy, which protects hearts by suppressing cellular levels of ROS and modulates mitochondrial intermediary metabolism and fatty acid utilization during fasting. Another one, Hydrogen sulfide is produced within the human body, and relaxes the vascular endothelium and smooth muscle cells, which is important to maintaining clean arteries as one age. It functions as an antioxidant and inhibits expression of pro-inflammatory factors, all of which imply an important role in aging and ageassociated diseases. The aim of this review is to find out the anti aging properties of SIRT3 and $\mathrm{H}_{2} \mathrm{~S}$ that can be used to extend the life span of human as a preventive and therapeutic agent.
\end{abstract}

Keywords: SIRT3, $\mathrm{H}_{2} \mathrm{~S}$, ROS, Aging

\section{Introduction}

The sirtuins are conserved genes that are found in bacteria to mammals. There are seven sirtuins genes (SIRT1-7) are found in the genome of mammal which share a highly conserved NAD+ binding catalytic domain [1]. Without considering the homology of sirtuins genes, they appear to have specific and individual biological functions due to their different substrates, sub cellular localization and patterns of expression. Among the sirtuin genes (SIRT1-7), SIRT3 was initially localized in mitochondria, but recent studies have identified that it is expressed in the nucleus as well [2]. SIRT3 has substrates in both nucleus and mitochondrion. In nucleus, the substrate of SIRT3 gene includes Histone 3 (H3), $\mathrm{H} 4$, and $\mathrm{Ku} 70$ and in mitochondrion, the substrate of this gene comprises acetyl-CoA-synthetase 2 [2,3]. On the other hand, in general, glutamate dehydrogenase, isocitrate dehydrogenase 2, Ku70, Foxo3a, electron transport chain complex I subunit NDUFA9 ATP synthase and succinate dehydrogenase were also reported to be SIRT3 substrates [4,5]. Human SIRT3 gene encodes a protein containing 399 amino acids where 25 amino acids mitochondrial localization sequence is present at its $\mathrm{N}$-terminus and the weight of this protein is approximately $44 \mathrm{kDa}$. This protein is synthesized as enzymatically inactive form in the mitochondrial inner membrane $[6,7]$. It is cleaved by matrix-processing peptidase to become a $28 \mathrm{kDa}$ enzymatically active mature form in the mitochondrial matrix [7]. Functionally, SIRT3 was associated to deacetylate and activate acetyl- CoA synthetase 2 (ACS2), an important mitochondrial enzyme involved in generating acetyl-CoA from acetate [8]. Aging is a multifaceted degenerative process but lifespan can be extended by single gene mutations [9]. Tissue-specific stem cells plays important role for self-renewal and persist throughout an organism's lifespan to repair and maintain tissues. But the self-renewal potential and differentiation capacity of stem cells become dysregulated with age [10]. This is because stem cell aging is believed to face cumulative cellular and genomic damages, 
resulting in permanent cell-cycle arrest, apoptosis, or senescence [10]. One of the major causes of cellular damage is the activity of Reactive Oxygen Species (ROS), a natural by-product of cellular respiration. ROS levels in stem cells increase dramatically with aging [11]. Deficient intracellular management of ROS affects on increased stem cell cycling and apoptosis, as well as negotiates self-renewal and differentiation, resembling essential aspects of aged stem cells [12]. Among the seven SIRT analogues, SIRT3 is the only member whose increased expression has been linked to the longevity of human. Polymorphism in the SIRT3 gene promoter that leads to gene activation has been found effective to extend life span of human [13]. In the modern time, $\mathrm{H}_{2} \mathrm{~S}$ has been gaining significant attentions as an important endogenous signaling molecule because of its significant effects on the cardiovascular and nervous systems. At the present time, a fact is rising that hydrogen sulfide slows aging by inhibiting free-radical reactions by activating SIRT1; an enzyme believed to be a regulator of lifespan. Besides, it is now thought that $\mathrm{H}_{2} \mathrm{~S}$ has interaction with a gene named klotho, which appears to have anti-aging activity [14].

\section{Sirt3 Activities}

\subsection{SIRT3 is Controlled in Skeletal Muscle by Exercise Training}

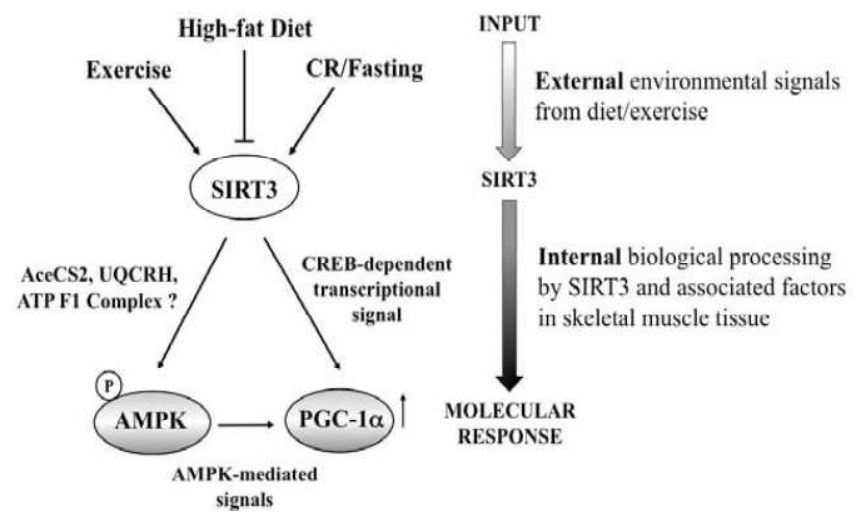

Figure 1. Schematic diagram of potential SIRT3 action in the skeletal myocyte. High fat diet suppressed SIRT3 expression and decrease SIRT3 protein level whereas exercise and fasting enhanced SIRT3 expression.

SIRT3 protein level is reduced by high-fat intake, while it is increased by short-term fasting or long-term nutrient deprivation and exercise training. It is assumed that SIRT tissue distribution pattern reflects SIRT3 mRNA [6]. Palacios et al. (2009) [15] assayed SIRT3 expression profile in vivo to compare the whole-body distribution of SIRT3. In case of muscle sample, it is found that SIRT3 protein levels were higher in the slow-twitch soleus muscle compared to the fasttwitch muscles like extensor digitorum longus and gastrocnemius. Further, a investigation driven by a specific antimouse SIRT3 polyclonal antibody, It is also found that SIRT3 protein, citrate synthase activity, a mitochondrial marker for exercise training were significantly higher in trained muscles than in the respective sedentary control group (Palacios et al., 2009)[15]. Collectively, these data represents that the upregulation of SIRT3 by exercise is an important and conserved molecular consequence of training

\subsection{SIRT3 Expression in Skeletal Muscle is Sensitive to Dietary Intake}

$\mathrm{CR}$ (caloric restriction) is known as a potential environmental method to extend lifespan in a number of organisms from yeast and nematodes to rodents but so far, the underlying molecular mechanisms by which this pathway acts remain largely unknown [16]. Palacios et al. (2009) [15] measured the SIRT3 levels in mice leg muscles after twelve months implication of either caloric restriction or add libitum cohorts. They found that SIRT3 protein levels in skeletal muscle were sensitive to diet and SIRT3 expression increases by fasting and caloric restriction, whether it was decreased by high fat diet. The caloric restriction practice also leads to phosphor activation of AMPK in muscle. They also found that the phosphorylation of both AMPK and CREB and the expression of PGC $1 \alpha$ are down regulated, suggesting that these key cellular factors may be important components of SIRT3 mediated biological signals in vivo.

\subsection{SIRT3 Regulates Mitochondrial Protein Synthesis}
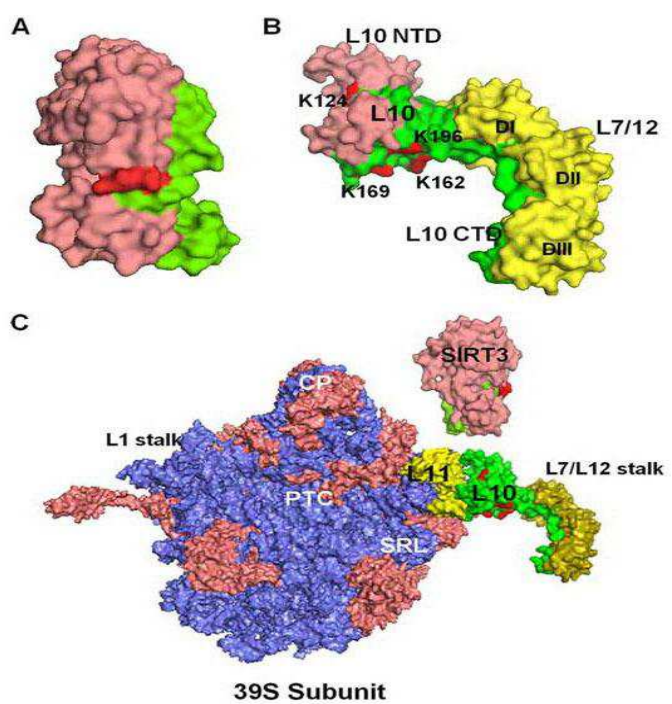

Figure 2. Structural models of the SIRT3 and MRPL10 interactions in the ribosomal L7/L12 stalk. A, crystal structure model of the human SIRT3 showing the MRP-L10 (green surface) interaction site and the Acecs 2 peptide at the active site (red). B, structure of the L10-L7/L12 complex from T. maritimi was used to model L7/L12 stalk in mitochondria. In the model, MRPL10 was colored pink and green, and the conserved Lys residues observed to be acetylated in bovine MRPL10 were colored red. The L7/L12 dimers (DI, DII, and DIII) were colored yellow. C, models of the human SIRT3 and T. maritimi L10-L7/L12 complex were used to represent their possible interactions with $55 S$ ribosomes using coordinates from the E. coli $50 S$ subunit. The 50 S ribosomal rRNAs, L10, and SIRT3 were colored blue, green, and pink, respectively. The other functional regions, such as peptidyltransferase center (PTC), central protuberance (CP), sarcin-ricin loop (SRL), L1, and L7/L12 stalks of the large subunit and ribosomal proteins (salmon) are labeled in the model. NTD, N-terminal domain; CTD, C-terminal domain [22]. 
Through the oxidative phosphorylation, mitochondria produce over $90 \%$ of the energy used by mammalian cells. Reversible acetylation leads to control major biological processes including mitochondrial energy metabolism [17, 18]. Here, the enzymes linked with the acetylation of mitochondrial proteins are not known but members of the class III histone deacetylases (sirtuins), SIRT3, SIRT4, and SIRT5, have been found to be inherent in mitochondria [19]. Mammalian mitochondrial ribosomal proteins are all nucleus-encoded, and some of them have been mapped to regions associated with disorders of mitochondrial energy metabolism [20]. Alterations in expression levels and mutations of these ribosomal proteins influence the mitochondrial protein synthesis, cell growth, and apoptosis [21]. Mitochondrial protein synthesis is regulated by reversible acetylation of MRPL10 and that the NAD dependent SIRT3 stimulates deacetylation of MRPL10, consequently regulating protein synthesis in mammalian mitochondria. Yang et al. 2010 [22] studied on Sirt3 Knockout Mice (mice in which the SIRT3 gene was targeted by gene trapping) mapped the acetylated Lys residues by tandem mass spectrometry and determined the role of these residues in acetylation of MRPL10 by site-directed mutagenesis. Furthermore, they found that the elevated acetylation of MRPL10 led to an increase in translational activity of mitochondrial ribosomes in SIRT3 in mice. In a similar manner, ectopic expression and knockdown of SIRT3 in $\mathrm{C} 2 \mathrm{C} 12$ cells resulted in the suppression and enhancement of mitochondrial protein synthesis, respectively. They proposed that the regulator of mitochondrial protein synthesis by the reversible acetylation of the mitochondrial ribosome and characterize MRPL10 as a novel substrate of the NADdependent deacetylase, SIRT3.

\subsection{SIRT3 Blocks the Cardiac Hypertrophic Response}

Cardiac hypertrophy is a common response of myocytes which is caused by variety of physiologic and pathologic stimuli. Cardiac hypertrophy may be occurred when mammalian cardiomyocytes failure their ability to divide soon after birth, then the only way for them to deal with a sustained enhance in workload is to undergo hypertrophy. At the time of hypertrophy, myocytes are growing and they connect sarcomeres and stimulate the expression of a group of genes, which are usually expressed during fetal heart development. These changes may be compensatory primarily to manage the raised workload on the heart. However, prolonged hypertrophy results in congestive heart failure and sudden death due to arrhythmias [23]. At the molecular level, hypertrophy of cardiomyocytes is thought as an outcome of imbalance between prohypertrophic and antihypertrophic factors and their downstream mechanisms control cell growth. Sundaresan et al. (2008) [3] examined the role of SIRT3 in murine cardiomyocytes. They observed that SIRT3 is a stressresponsive deacetylase and its high expression protects myocytes from genotoxic and oxidative stress-mediated cell death. They also found that mouse SIRT3 was expressed in two forms, like human SIRT3. They are 44-kDa long form and
$28-\mathrm{kDa}$ short form. The long form was located in the mitochondria, nucleus, and cytoplasm; the short form is localized exclusively in the mitochondria of cardiomyocytes. During stress, SIRT3 levels are raised not only in mitochondria but also in the nuclei of cardiomyocytes. They identified Ku70 (a nuclear protein, as a deacetylase target of SIRT3) as a new target of SIRT3. SIRT3 physically binds to $\mathrm{Ku} 70$ and deacetylates it, and this promotes interaction of $\mathrm{Ku} 70$ with the proapoptotic protein Bax. Thus, under stress conditions, increased expression of SIRT3 protects cardiomyocytes, in part by hindering the translocation of Bax to mitochondria. This study found a vital role of SIRT3 on survival of cardiomyocytes in stress situations. On the different study Sundaresan et al. (2009) [24] used Sirt3-deficient and Sirt3overexpressing $\mathrm{Tg}$ mice for analyzing the cardiac hypertrophic response and the involvement of Sirt3 In preliminary cultures of cardiomyocytes, they observed SIRT3 blocked cardiac hypertrophy by activating the forkhead box O3a-dependent (Foxo3a-dependent) antioxidant encoding genes manganese superoxide dismutase (MnSOD) and catalase (Cat); thereby reducing cellular levels of ROS. Decreased ROS levels helped to reduce Ras activation and downstream signaling through the MAPK/ERK and PI3K/Akt pathways. This resulted is repressed activity of transcription factors, specifically GATA4 and NFAT, and translation factors, specifically eukaryotic initiation factor 4E (elf4E) and S6 ribosomal protein (S6P), which are connected in the development of cardiac hypertrophy. Their results reveal that SIRT3 is an endogenous negative regulator of cardiac hypertrophy, which protects hearts by decreasing cellular levels of ROS.

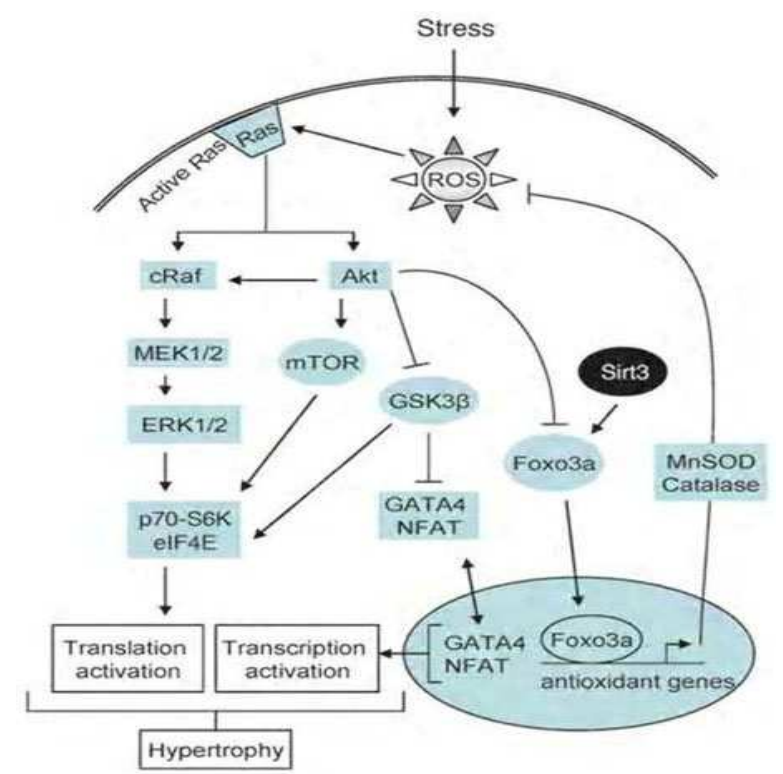

Figure 3. Scheme illustrating signaling pathways modified by SIRT3 to block the cardiac hypertrophic response. Sirt3 levels are increased during stress of cardiomyocytes, which deacetylates Foxo3a and traps it inside the nucleus to elevate the transcription of Foxo-dependent antioxidant genes, MnSOD and Cat. Raised expression of MnSOD and catalase suppresses ROS levels generated by stress stimuli. Because ROS is the second messenger of hypertrophic signaling pathways, suppression of ROS levels shuts down major signaling pathways involved in activation of transcription and translation events contributing to the cardiac hypertrophic response. 


\subsection{SIRT3 Regulates Fatty Acid Oxidation}

Proteomics analysis of mitochondrial proteins showed that the acetylation levels of numerous mitochondrial proteins changed during fasting [17]. The dependence of SIRT3 enzymatic activity on NAD+ suggests that SIRT3 could serve as a metabolic sensor and combine the energy status of the cell with the level of acetylation of mitochondrial protein [25]. Hirschey et al. (2010) [26] investigated on mice and reported that SIRT3 expression was positively regulated during fasting in liver and brown adipose tissues. Livers from mice lacking SIRT3 was shown higher levels of fatty acid oxidation intermediate products and triglycerides. When fasting was associated, decreased levels of fatty acid oxidation was observed. Mass spectrometry analysis of mitochondrial proteins also revealed that long-chain acyl CoA dehydrogenase (LCAD) was hyperacetylated at lysine 42 in the absence of SIRT3. LCAD was deacetylated in wildtype mice under fasted conditions and by SIRT3 in vitro and in vivo, and hyperacetylation of LCAD declined its enzymatic activity. Moreover, which mice lacking of SIRT3 expressed fatty acid oxidation disorders during fasting including reduced ATP levels and intolerance to cold exposure. These findings recognize acetylation as a novel regulatory mechanism for mitochondrial fatty acid oxidation and show that SIRT3 modulates mitochondrial intermediary metabolism and fatty acid utilization during fasting [26].

\subsection{The Directs Enzymatic Detoxification Activity of SIRT3}

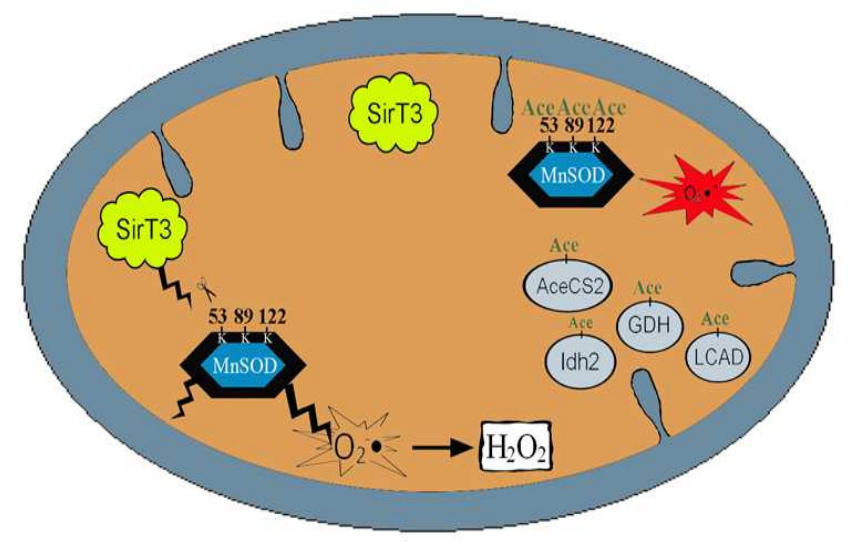

Figure 4. Proposed model figure describing Sirt3 acetylation and subsequent regulation of MnSOD detoxification enzymatic activity. Sirt3 is localized into the inner mitochondrial membrane and appears to be activated by agents that induce oxidative stress, such as ionizing radiation, or changes in cellular nutrient status, such as caloric restriction or fasting. Sirt3 has also been shown to regulate the activity of other mitochondrial proteins including Acetyl CoA synthetase 2 (AceCS2), glutamate dehydrogenase $(G D H)$, long chain acyl CoA dehydrogenase (LCAD), and isocitrate dehydrogenase 2 (Idh2)[32].

Manganese superoxide dismutase is one of the fundamental mitochondrial antioxidants among a series of detoxification enzymes that neutralizes the highly reactive superoxide ions to less reactive hydrogen peroxide $\left(\mathrm{H}_{2} \mathrm{O}_{2}\right)$ followed by its immediate conversion to $\mathrm{H}_{2} \mathrm{O}$ by catalase and other peroxidases in the mitochondrial matrix [27].
Superoxide is the incidental product of electron transport chains I and III while they perform inefficiently, and arises as a primary damaging reagent for mtDNA and other mitochondrial macromolecules $[28,29]$. ROS is the sequential reduction from $\mathrm{O} 2$, include superoxide $\left(\mathrm{O} 2^{-}\right)$, hydrogen peroxide ( $\mathrm{H} 2 \mathrm{O} 2)$, hydroxyl radical $(. \mathrm{OH})$, and organic peroxides, that are normally made during respiration [30]. Low amounts of ROS are natural side products of various electron transfer reactions and easily tolerated by the cell whereas abnormally high levels of ROS from any number of possible sources induces oxidative stress and can damage cells by peroxidizing lipids, and disrupting proteins and nucleic acids [31]. A basic result in biology is that mitochondrial function changes significantly with age, expressing a link between the cellular longevity and mitochondrial metabolism homeostasis. Altered ROS levels are found in multiple ages' related illnesses including carcinogenesis, neurodegenerative, fatty liver, insulin resistance, and cardiac disease etc. It has recently been revealed that manganese superoxide dismutase enzymatic activity is regulated by the reversible acetylation of specific lysine(s) in the protein. These results, propose for the first time, that the mitochondria contain bidirectional posttranslational signaling networks, similar to that found in the cytoplasm and nucleus, and that changes in lysine acetylation alter manganese superoxide dismutase enzymatic activity. Besides, these new results divulge that the mitochondrial antiaging sensing protein SIRT3 responds to changes in mitochondrial nutrient and redox status. That is why, this phenomenon leads to alter the enzymatic activity of manganese superoxide dismutase that adjusts and maintains ROS levels as well as metabolic homeostatic poise [32].

\subsection{SIRT3 Modulates Hepatic Susceptibility to Lipotoxicity}

Bao et al. (2010) [33] investigated the regulation of SIRT3 in the electron transfer chain and estimated the role of SIRT3 in hepatic lipotoxic stress on mouse. SIRT3 reduced HepG2 cells which was responsible to diffuse disruption in mitochondrial electron transfer chain which leads to a concurrent reduction in the mitochondrial membrane potential, and excess basal reactive oxygen species levels. These phenotypes predispose increased lipotoxic hepatic susceptibility that they were evaluated the expression of SIRT3 in murine liver following chronic high-fat feeding. In this nutrient-excess model SIRT3 transcript and protein levels were down regulated in parallel with increased hepatic fat storage and oxidative stress. Palmitate was used to investigate lipotoxic susceptibility in SIRT3 knockout mouse primary hepatocytes and SIRT3 siRNA depleted HepG2 cells. When SIRT3 was not present, palmitate raised reactive oxygen species and enhanced hepatocyte cell death. Reconstitution of SIRT3 levels and treatment with Nacetylcysteine improved these adverse effects [33]. SIRT3 functions ameliorate hepatic lipotoxicity, although paradoxically exposure to high-fat down regulates this adaptive program in the liver. This SIRT3-dependent lipotoxic susceptibility is possibly modulated, to some extent, 
by SIRT3 mediated control of electron transfer chain.

\subsection{SIRT3 Regulates Ketone Body Production}

At the time of low nutrient conditions, organisms depend on adaptive metabolic mechanisms to maintain energy homeostasis. In the transition, from the fed to the fasted state, carbohydrate utilization and fatty acid synthesis stop in the liver, and fatty acid oxidation and ketogenesis are introduced [34]. Acetyl-CoA produced from fatty acid oxidation is diverted away from the tricarboxylic acid (TCA) cycle and converted into acetoacetate, $\beta$-hydroxybutyrate, and acetone (ketone bodies) through ketogenesis in the mitochondria [35]. Specific tissues, such as the brain use acetoacetate and $\beta$ hydroxybutyrate to spare glucose when glucose levels are low. Shimazu et al. (2010) [36] identified mitochondrial 3-hydroxy3-methylglutaryl CoA synthase 2 (HMGCS2) as an acetylated protein in a proteomics survey and a possible target of SIRT3 in hepatic mitochondria of Sirt3-/- (SIRT3KO) mice. When HMGCS2 was the rate-limiting step $\beta$-hydroxybutyrate synthesized at lysines 310,447 , and 473 in the absence of SIRT3. However, HMGCS2 was deacetylated by SIRT3 in response to fasting in wild-type mice, but not in SIRT3KO mice. On the other hand, HMGCS2 was deacetylated in vitro when incubated with SIRT3 and in vivo by overexpression of SIRT3. Deacetylation of HMGCS2 lysines 310, 447, and 473 by incubation with wild-type SIRT3 or by mutation to arginine enhanced its enzymatic activity. Molecular dynamics simulations represented that silico deacetylation of these three lysines causes conformational change of HMGCS2 near the active site. Mice which were lacking SIRT3 showed reduced $\beta$ - hydroxybutyrate levels during fasting. Finally Shimazu et al. (2010) [36] noted that SIRT3 regulated ketone body production during fasting and provided molecular insight that how protein acetylation could regulate enzymatic activity.

\subsection{SIRT3 Opposes Reprogramming of Cancer Cell Metabolism}

Otto Warburg first reported in the 1920s that cancer cells undergo glycolysis even in the existence of ample oxygen [37]. This use of aerobic glycolysis, called the Warburg effect, has appeared as a metabolic characteristic of many cancers. Accruing evidence proposes that aerobic glycolysis is used to support the rapid proliferation of tumor cells. Increased catabolism of glucose contributes to the raw materials needed to synthesize the nucleotides, amino acids and lipids necessary for cellular proliferation and can support as a distinct growth advantage for cells with elevated aerobic glycolysis [38]. Essential up regulation of aerobic glycolysis can also make a survival advantage for tumor cells because limitations in tumor vascularization result in periods of intermittent hypoxia that requires a cell to depend on glycolysis [39].On the other hand, the transition from premalignant lesions to invasive cancer is often accompanied by enhanced tumor glucose uptake [39]. Metabolic reprogramming in cancer cells is controlled by several oncogenic signs, including the PI3K/Akt, Myc or hypoxia- inducible transcription factor (HIF) pathways that serve to increase glucose uptake, glycolysis, angiogenesis and stress resistance $[40,41,42]$. Either gain or loss of function mutations in tricarboxylic acid (TCA) cycle enzymes regulate HIF1 activity and promote carcinogenesis [43, 44]. For this reason, identification of new mitochondrial regulators could provide important insight on the contribution of altered metabolism to tumorigenesis. Finley et al. (2011) [45] revealed that the mitochondrial NAD dependent deacetylase SIRT3 was a crucial regulator of the Warburg effect. Mechanistically, SIRT3 mediated the metabolic reprogramming by destabilizing hypoxia-inducible factor-1 $\alpha$ (HIF1 $\alpha)$, a transcription factor that controls glycolytic gene expression. Loss of SIRT3 increases reactive oxygen species production, leading to HIF1 $\alpha$ stabilization. SIRT3 expression is declined in human breast cancers, and its loss correlates with the up regulation of HIF1 $\alpha$ target genes. Finally, Finley et al. (2011) [45] reported that SIRT3 over expression suppress glycolysis and proliferation of breast cancer cells, providing a metabolic mechanism for tumor suppression. In 2011, Schumacker [46] revealed that genetic loss of the deacetylase SIRT3 leads to metabolic reprogramming toward glycolysis. This shift is occurred by an increase of cellular reactive oxygen species (ROS) generation that increases HIF- $\alpha$ stabilization and HIF dependent gene expression, thereby driving the tumor formation.

\subsection{SIRT3 Deficiency Promotes Development of Metabolic Syndrome}

Acetylation is known as an important metabolic regulatory post-translational protein modification but so far, the metabolic consequence of mitochondrial protein hyperacetylation is unknown. In a experiment, it was found that high-fat diet (HFD) feeding leads to hepatic mitochondrial protein hyperacetylation in mice and down regulate the major mitochondrial protein deacetylase SIRT3 [47]. Mice lacking SIRT3 (SIRT3KO) placed on a HFD shown accelerated obesity, insulin resistance, hyperlipidemia, and steatohepatitis compared to wild-type (wt) mice. The lipogenic enzyme stearoyl-CoA desaturase 1 is found in SIRT3KO mice, and its deletion liberates both wt and SIRT3KO mice from HFD-induced hepatic steatosis and insulin resistance. They further identified a single nucleotide polymorphism in the human SIRT3 gene that is suggestive of a genetic association with the metabolic syndrome. This polymorphism encodes a point-mutation in the SIRT3 protein, which reduces its overall enzymatic efficiency. Finally, Hirschey et al. (2011) [47] showed that loss of SIRT3 and dysregulation of mitochondrial protein acetylation contributed to the metabolic syndrome.

\subsection{SIRT3 Suppresses Hypoxia Inducible Factor 1a and Tumor Growth}

Oncogenic transformation is regulated by a variety of cellular changes, including changes in metabolism. Though it is not clear whether the alterations in metabolism are a cause 
or an affect of transformation, but they can help cancer cells to survive various stresses such as hypoxia and glucose limitation. Some of these changes are activated by the transcription factor hypoxia inducible factor 1a (HIF-1a). HIF-1a is a heterodimer of two basic helix-loop-helix/PAS proteins, HIF-1a and the aryl hydrocarbon nuclear translocator (ARNT or HIF-1b) [48]. An alternate isoform, HIF-2a has both overlapping and non-redundant functions with HIF1a [49]. Both HIFa subunits and ARNT are expressed together; however, the alpha subunit is labile in conditions of normal oxygen $(5-21 \%$ O2). Under hypoxic conditions $(0.5-$ $5 \% \mathrm{O} 2$ ) the HIFa subunit is stabilized, dimerizes with ARNT, translocates to the nucleus, and subsequently binds to HIF response elements (HRE) within target genes. Among HIF-1a, transcription targets are genes linked in glucose metabolism, angiogenesis and metastasis [50] thereby tightly linking HIF1a-mediated transcription to tumorigenesis. Bell et al. (2011) [51] found that the mitochondrial sirtuin, SIRT3, acts as a tumor suppressor via its ability to suppress reactive oxygen species (ROS) and regulate hypoxia inducible factor 1a (HIF1a). They also observed that primary mouse embryo fibroblasts (MEFs) or tumor cell lines expressing SIRT3 short-hairpin RNA exhibited a greater potential to proliferate and augmented HIF-1a protein stabilization and transcriptional activity in hypoxic conditions. Reduced SIRT3 found to increase tumorigenesis in xenograft models and this effect could be abolished by the introduction of antioxidant Nacetyl cysteine to mice. When SIRT3 was over expressed, they found decrease of tumorigenesis in xenografts, even the induction of sirtuin after tumor initiation [51]. Their study suggested that SIRT3 acted to suppress the growth of tumors, at least to a certain extent through its ability to suppress ROS and HIF-1a.

\subsection{Neuronal SIRT3 Protects Against Excitotoxic Injury}

Continuous provider of energy is crucial for neuron survival due to the requirement for large amounts of energy for high metabolic processes coupled with an inability to store energy [52]. Neurons are highly susceptible to insults that authorize to energy depletion, such as oxidative stress, excitotoxicity, and DNA damage [53]. As a vital factor for energy metabolism, nicotinamide adenine dinucleotide (NAD) has managed a considerable attention. NAD is an important molecule playing a crucial role in energy metabolism, cellular redox reaction, and mitochondrial function. Recent studies have noted that maintaining intracellular NAD is essential in promoting cell survival in various types of diseases, including axonal degeneration, multiple sclerosis (MS), cerebral ischemia, and cardiac hypertrophy [54,55]. Loss of NAD declines the ability of NAD dependent cell survival factors to carry out energy dependent processes, leading to cell death. PARP-1, a major NAD metabolizing enzyme, hydrolyzes NAD to nicotinamide and produces poly (ADP) ribose polymers (PAR) upon activation under pathological condition, and brings to severe impairment of energy metabolism with almost complete depletion of cytosolic and nuclear NAD [56,57]. Genotoxic injury, overstimulation of N-methyl-D-aspartate (NMDA) receptors, or oxidative stress has been reported to activate PARP-1 in neurons $[58,59]$. PARP-1 mediated NAD depletion produces mitochondrial permeability transition (MPT) causing cell death under DNA damaging injury $[60,61]$. To induce excitotoxic injury, Kim et al. (2011) [62] exposed primary cultured mouse cortical neurons to NMDA (30 mM). NMDA induced a rapid decrease of cytoplasmic NAD (but not mitochondrial NAD) in neurons through poly (ADP-ribose) polymerase-1 (PARP-1) activation. Mitochondrial SIRT3 was increased following PARP-1 mediated NAD depletion, which was reversed by either inhibition of PARP-1 or exogenous NAD. They found that huge amount of reactive oxygen species (ROS) were produced under NAD depleted condition and this happening mediated the increase of mitochondrial SIRT3.By transfecting primary neurons with a Sirt3 over expressing plasmid or Sirt3 siRNA, they showed that Sirt3 is required for neuroprotection against excitotoxicity. Their study proposed for the first time that mitochondrial Sirt3 acts as a prosurvival factor playing an essential role to protect neurons under excitotoxic injury.

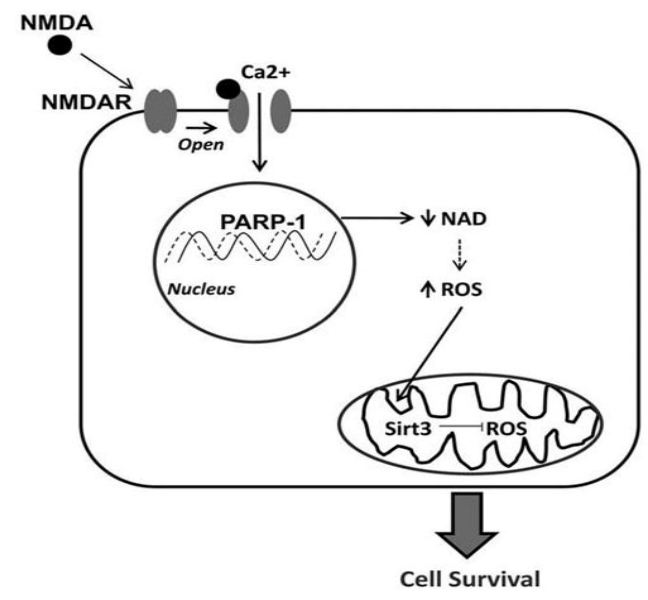

Figure 5. Schematic Diagram of NMDA-induced increase in mitochondrial Sirt3. The proposed mechanism representing the action of Sirt 3 in protecting against NMDA-induced excitotoxic injury. NMDA binds to the NMDA receptor which opens the ion channel and allows for calcium (Ca2+) influx into the cell and promotes oxidative stress. Oxidative DNA damage activates PARP-1 and decreases NAD, leading to an increase in ROS production due to mitochondrial failure. The increase in ROS promotes an increase in mitochondrial Sirt3. Mitochondrial Sirt3 levels were elevated in NMDAtreated neurons following PARP-1 mediated NAD depletion. Overexpression of Sirt3 prevented the increase in ROS production and neuronal death, whereas knock down of Sirt3 exacerbated neuronal excitotoxic injury [62].

\section{Hydrogen Sulfide}

\subsection{Nutrition, Metabolism and Homeostasis}

The major dietary sources of sulfur compounds in human nutrition are inorganic sulfates of drinking water and proteins which are derived from plants and animals. Sulfur is present in only two of the twenty amino acids and these two amino acids are called sulfur-containing amino acids (SAAs), namely methionine and cysteine. Dietary excess of cysteine and methionine is reserved as GSH (a thiolic antioxidant 
tripeptide) pools or converted to taurine or oxidized to sulfate In fact, the availability of cysteine appears to be the rate limiting factor for GSH biosynthesis from glutamate, glycine, and cysteine [63]. The sulfane sulfur pool performs an essential function in the brain, upon neuron excitation the bound sulfane sulfur releases $\mathrm{H}_{2} \mathrm{~S}$ [64]. Homeostatic controlling of cysteine and GSH pools declines with age and this declination appearing in men at a younger age than in women.

\subsection{Inflammation and Immunity}

$\mathrm{H}_{2} \mathrm{~S}$ can control inflammation and cell death, probably by exerting its valuable effects on ATP-sensitive $\mathrm{K}+$ channels [65] inhibition of activation of NF-Jb and p38 MAPK, scavenging of oxidants, up regulation of intracellular cAMP, and inhibition of caspase-3 cleavage [66]. Chronic inflammation is linked in some of the most common human diseases such as rheumatoid arthritis, tuberculosis, asthma, inflammatory bowel disease, vasculitis, and Crohn's disease. Chronic inflammation is an influential factor in type II diabetes, cardiovascular disease, and tumor development $[67,68]$.

\subsection{Cytoprotection and Pharmacological Conditioning}

$\mathrm{H}_{2} \mathrm{~S}$ strictly influences the body's redox status through various mechanisms, such as enhancing GSH levels in the cytosol, mitochondria, and nucleus of cells, elevating the GSH/GSSG ratio, activating the reperfusion injury salvage kinase (RISK) pathway with up regulation of protective heatshock proteins. $\mathrm{H}_{2} \mathrm{~S}$ also acts as a switch of $\mathrm{Nrf} 2$ nuclear translocation, resulting persistent activation of the antioxidant responsive elements (AREs) of antioxidant genes and over expression of antioxidant and phase II enzymes [69] $\mathrm{H}_{2} \mathrm{~S}$ is not only responsible for anti-apoptotic and antiinflammatory effects but also stands for anti-nociceptive and blood pressure lowering affect by activating KATP channels [65]. The cardio protective effect of $\mathrm{H}_{2} \mathrm{~S}$ also responsible to the activation of cardiac extracellular signal regulated- kinase and Akt pathways [65].

\subsection{Nervous System}

1-Methyl-4-phenyl-1,2,3,6-tetrahydropyridine (MPTP) is a neurotoxin that can influence Parkinson's disease like symptoms and biochemical changes in animals and humans. Inhaled $\mathrm{H}_{2} \mathrm{~S}$ has been shown by Kida et al. to prevent MPTPinduced movement disorder, neuron degeneration, and neuron apoptosis and gliosis in mice [70]. These effects were assigned to up regulation of genes encoding antiinflammatory and antioxidant proteins, including heme oxygenase-1 (HO-1) and glutamate-cysteine ligase.

\subsection{Liver and Kidneys}

$\mathrm{H}_{2} \mathrm{~S}$ regulates intrahepatic blood flow (microcirculation) in the normal and cirrhotic liver [71] with insufficient making of $\mathrm{H}_{2} \mathrm{~S}$ in the cirrhotic liver and down regulation of $\mathrm{H}_{2} \mathrm{~S}$ producing enzymes in kidney and liver of patients with chronic kidney disease [72].Administration of $\mathrm{H}_{2} \mathrm{~S}$ has been observed to protect the liver and kidneys from ischemia reperfusion damage. In kidneys, $\mathrm{H}_{2} \mathrm{~S}$ has been found to be beneficial for the prevention or treatment of diabetic kidney disease via improving renal glycative injury [73] increasing renal blood flow, glomerular filtration rate, urinary sodium excretion and ameliorating hyperhomocysteinemia associated chronic renal failure [74]. In the liver, $\mathrm{H}_{2} \mathrm{~S}$ effectively reduces stress-mediated liver injury and hepatic mitochondrial dysfunction in acutely ethanol-exposed mice, and markedly enhances acetaminophen-induced hepatotoxicity in mice [75].

\section{6. $\mathrm{H}_{2} \mathrm{~S}$ as an Antioxidant and Free Radical Scavenger}

At $37^{\circ} \mathrm{C}$ and physiological fluid $\mathrm{pH}(\mathrm{pH} 7.4)$, about $80 \%$ of the $\mathrm{H}_{2} \mathrm{~S}$ molecules dissociate to produce HS- (hydrosulfide anion), which is therefore the predominant sulfur containing species in extracellular fluids and plasma, whereas within the cell ( $\mathrm{pH}$ about 7.2) the amounts of $\mathrm{H}_{2} \mathrm{~S}$ and HS- are nearly equal [76]. Hydrosulfide anions are powerful reductants that capable of suppressing free radicals by transferring hydrogen atom or by transferring single electron usually at a diffusioncontrolled rate. The oxidation of hydrosulfide anions by biochemically relevant two-electron oxidants (e.g., hypochlorous acid and hydrogen peroxide) forms initially hydrogen disulfide $\left(\mathrm{H}_{2} \mathrm{~S}_{2}\right.$, also known as disulfane) which is also a highly reactive oxidizing agent has a ability to regenerate $\mathrm{H}_{2} \mathrm{~S}$ by reaction with a thiol or by disproportionate [77]. $\mathrm{H}_{2} \mathrm{~S}$ readily scavenges ROS and RNS containing hypochlorous acid, hydrogen peroxide, lipid hydroperoxides, $\mathrm{O}_{2}$ and peroxynitrite [78]. It has also a capability of scavenging the triplet state of riboflavin. However, in the presence of molecular oxygen (dioxygen form) oxidation of $\mathrm{H}_{2} \mathrm{~S}$ generates free radicals [79].

Table 1. Anticancer Effect of $\mathrm{H}_{2} \mathrm{~S}$

\begin{tabular}{lll}
\hline Effect & Mediators involved & References \\
\hline Enhanced immunocompetence & GSH, Taurine & {$[63,80,81]$} \\
Inhibition of procarcinogen of activated by oxidases & Nrf2 & {$[82]$} \\
Epigenetic silencing of protooncogen & SAM & {$[83,68]$} \\
Epigenetic reactivation of tumor suppressor genes & HDAC & {$[84]$} \\
DNA protection/repair & GSH, Trx & {$[85,78]$} \\
Abolishment of chronic inflammation & GSH & {$[86,63]$} \\
Prooxidant/proapoptotic redlining & Sulfane sulfur, ROS & {$[68]$} \\
Antimetastatic effect & E-Cadherin & {$[87,88]$} \\
\hline
\end{tabular}




\section{Conclusion}

Desulfhydration of cysteine is the main source of $\mathrm{H}_{2} \mathrm{~S}$ in mammals and is catalyzed by the trans-sulfuration pathway enzymes cystathionine beta-synthase (CBS), cystathionine gamma-lyase (CSE) and 3-mercaptopyruvate sulfurtransferase (3- MST). Endogenous $\mathrm{H}_{2} \mathrm{~S}$ has been found wanting in an animal model of Parkinson's disease and is found to be depressed in the brains of patients with Alzheimer's disease. An abundance of experimental evidence proposes that $\mathrm{H}_{2} \mathrm{~S}$ plays a vital role in normal physiology and pathophysiology. Therefore, many therapeutic targets exist for $\mathrm{H}_{2} \mathrm{~S}$ therapy, including cancer, heart failure, organ transplant, peripheral artery disease, inflammatory bowel disease, Alzheimer's disease, acute myocardial infarction (MI), stroke, atherosclerosis, hypertension, erectile dysfunction, metabolic syndrome, diabetes, and thrombosis. It also may play a wide-ranging role in staving off aging. On the other hand, SIRT3 has been found to link with longevity in men and aberrant expression of this sirtuin correlates with node-positive breast cancer in clinical biopsies from women, suggesting that SIRT3 serves as an important diagnostic and therapeutic target in human health/aging and disease, affecting men and women in unique ways. So, the conclusion that can be drawn from this review work is that, SIRT3 and $\mathrm{H}_{2} \mathrm{~S}$ may be used as an anti-aging therapeutic agent.

\section{References}

[1] Polito L., Kehoe PG., Forloni G., Albani D. (2010) The molecular genetics of sirtuins: association with humanlongevity and age-related diseases. Int J Mol Epidemiol Genet 1(3):214-225.

[2] Scher MB., Vaquero A., Reinberg D. (2007) SirT3 is a nuclear NAD+-dependent histone deacetylase that translocates to the mitochondria upon cellular stress. Genes Dev. 21:920-928.

[3] Sundaresan NR., Samant SA., Pillai VB., Rajamohan SB., Gupta MP. (2008) SIRT3 Is a Stress-Responsive Deacetylase in Cardiomyocytes That Protects Cells from Stress-Mediated Cell Death by Deacetylation of Ku70. Molecular and Cellular Biology. 28(20):6384-6401.

[4] Jacobs KM., Pennington JD., Bisht KS., Aykin-Burns N., Kim HS., Mishra M., Sun L., Nguyen P., Ahn BH., Leclerc J., Deng CX., Spitz DR., Gius D. (2008) SIRT3 interacts with the daf16 homolog FOXO3a in the mitochondria, as well as increases FOXO3a dependent gene expression. Int J Biol Sci. 4:291299.

[5] Cimen H., Han MJ., Yang Y., Tong Q., Koc H., Koc EC. (2009) Regulation of succinate dehydrogenase activity by SIRT3 in mammalian mitochondria. Biochemistry. 49:304-311.

[6] Onyango P., Celic I., McCaffery JM., Boeke JD., Feinberg AP. (2002) SIRT3, a human SIR2 homologue, is an NADdependent deacetylase localized to mitochondria. Proc Natl Acad Sci USA. 99:13653-13658.
[7] Schwer B., North BJ., Frye RA., Ott M., Verdin E. (2002) The human silent information regulator (Sir)2 homologue hSIRT3 is a mitochondrial nicotinamide adenine dinucleotidedependent deacetylase. J Cell Biol. 158:647-657.

[8] Hallows WC., Lee S., Denu JM. (2006) Sirtuins deacetylate and activate mammalian acetyl-CoA synthetases. Proc Natl Acad Sci USA. 103:10230-10235.

[9] Kenyon CJ. (2010) The genetics of ageing. Nature 464:504512 .

[10] Sahin E., Depinho RA. (2010) Linking functional decline of telomeres, mitochondria and stem cells during ageing. Nature 464:520-528.

[11] Ito K., Hirao A., Arai F., Takubo K., Matsuoka S., Miyamoto K., Ohmura M., Naka K., Hosokawa K., Ikeda Y., Suda T. (2006) Reactive oxygenspecies act through p38 MAPK to limit the lifespan of hematopoietic stem cells. Nat. Med. $12: 446-451$.

[12] Paik JH., Ding Z., Narurkar R., Ramkissoon S., Muller F., Kamoun WS., Chae SS., Zheng H., Ying H., Mahoney J., Hiller D., Jiang S., Protopopov A., Wong WH., Chin L., Ligon KL., DePinho RA. (2009) FoxOs cooperatively regulate diverse pathways governing neural stem cell homeostasis. Cell Stem Cell. 5:540-553.

[13] Bellizzi D., Rose G., Cavalcante P., Covello G., Dato S., De Rango F., Greco V., Maggiolini M., Feraco E., Mari V., Franceschi C., Passarino G., De Benedictis G. (2005) A novel VNTR enhancer within the SIRT3 gene, a human homologue of SIR2, is associated with survival at oldest ages. Genomics. $85: 258-263$.

[14] Zhang Y., Tang ZH., Ren Z., Qu SL., Liu MH., Liu LS., Jiang ZS. (2013) Hydrogen sulfide: the next potent preventive and therapeutic agent in aging and age-associated diseases. Mol. Cell. Bio. 33(6):1104-13.

[15] Palacios OM., Carmona JJ., Michan S., Chen KY., Manabe Y., Ward JL., Goodyear LJ., Tong Q.(2009) Diet and exercise signals regulate SIRT3 and activate AMPK and PGC- $1 \alpha$ in skeletal muscle. Aging. 1(9):771-783.

[16] Guarente L., Picard F. (2005) Calorie restriction the SIR2 connection. Cell. 120:473-482.

[17] Kim SC., Sprung, R., Chen Y., Xu Y., Ball H., Pei J., Cheng T., Kho Y., Xiao H., Xiao L.,Grishin N. V., White M., Yang XJ., Zhao Y. (2006) Substrate and functional diversity of lysine acetylation revealed by a proteomics survey.Mol. Cell. 23:607-618.

[18] Gerhart-Hines Z., Rodgers JT., Bare O., Lerin C., Kim SH., Mostoslavsky R., Alt FW., Wu Z., Puigserver P. (2007) Metabolic control of muscle mitochondrial function and fatty acid oxidation through SIRT1/PGC-1alpha.EMBO J. 26:19131923.

[19] Schwer B., Bunkenborg J., Verdin RO., Andersen JS., Verdin E. (2006) Reversible lysine acetylation controls the activity of the mitochondrial enzyme acetyl-CoA synthetase 2. Proc Natl Acad Sci U S A. 103:10224-10229.

[20] O'Brien TW., O'Brien BJ., Norman RA. (2005) Nuclear MRP genes and mitochondrial disease. Gene. 354:147-151. 
[21] Miller JL., Koc, H., and Koc, E. C. (2008).Identification of phosphorylation sites in mammalian mitochondrial ribosomal protein DAP3.Protein Sci. 17, 251-260.

[22] Yang Y., Cimen H., Han M-J., Shi T., Deng J-H., Koc H., Palacios OM., Montier L., Bai Y., Tong Q., Koc EC. (2010) NAD-dependent Deacetylase SIRT3 Regulates Mitochondrial Protein Synthesis by Deacetylation of the Ribosomal Protein MRPL10. Journal of Biological Chemistry. 285 (10):74177429.

[23] Frey N., Katus HA., Olson EN., Hill JA. (2004) Hypertrophy of the heart: a new therapeutic target? Circulation. 109:1580 1589.

[24] Sundaresan NR., Gupta M., Kim G., Rajamohan SB., Isbatan A., Gupta MP. (2009) Sirt3 blocks the cardiac hypertrophic response by augmenting Foxo3a-dependent antioxidant defense mechanisms in mice. J. Clin. Invest. 119:2758-2771.

[25] Guarente L. (2006) Sirtuins as potential targets for metabolic syndrome. Nature. 444:868-874.

[26] Hirschey MD., Shimazu T., Goetzman E., Jing E., Schwer B., Lombard DB., Grueter CA., Harris C., Biddinger S., Ilkayeva OR., Stevens RD., Li Y., Saha AK., Ruderman NB., Bain JR., Newgard CB., Farese Jr RV., Alt FW., Kahn CR., Verdin E. (2010) SIRT3 regulates fatty acid oxidation via reversible enzyme Deacetylation. Nature. 464(7285):121-125.

[27] Oberley LW., Oberley TD. (1988) Role of antioxidant enzymes in cell immortalization and transformation. Mol Cell Biochem. 84:147-153.

[28] Lanza IR., Short DK., Short KR., Raghavakaimal S., Basu R., Joyner MJ., McConnell JP., Nair KS. (2008) Endurance exercise as a countermeasure for aging. Diabetes. 57:29332942.

[29] Henderson JR., Swalwell H., Boulton S., Manning P., McNeil CJ., Birch-Machin MA. (2009) Direct, real-time monitoring of superoxide generation in isolated mitochondria. Free Radic Res. 43:796-802.

[30] Spitz DR., Adams DT., Sherman CM., Roberts RJ. (1992) Mechanisms of cellular resistance to hydrogen peroxide, hyperoxia, and 4hydroxy 2 nonenal toxicity: the significance of increased catalase activity in $\mathrm{H}_{2} \mathrm{O}_{2}$ resistant fibroblasts. Arch Biochem Biophys. 292:221-227.

[31] Sies H. (1991) Oxidative stress: from basic research to clinical application. Am J Med. 91:31S-38S.

[32] Ozden O., Park S-H., Kim H-S., Jiang H., Coleman MC., Spitz DR., Gius D. (2011) Acetylation of MnSOD directs enzymatic activity responding to cellular nutrient status or oxidative stress. Aging. 3:2

[33] Bao J., Scott I., Lu Z., Pang L., Dimond CC., Gius D., Sack MN. (2010) SIRT3 is regulated by nutrient excess and modulates hepatic susceptibility to lipotoxicity. Free Radic Biol Med. 49(7): 1230-1237.

[34] McGarry JD., Foster DW. (1980) Regulation of hepatic fatty acid oxidation and ketone body production. Annu. Rev. Biochem. 49:395-420.

[35] Laffel L. (1999) Ketone bodies: a review of physiology, pathophysiology and application of monitoring to diabetes. Diabetes Metab. Res. Rev.15:412-426.
[36] Shimazu T., Hirschey MD., Hua L., Dittenhafer-Reed KE., Schwer B., Lombard DB., Li Y., Bunkenborg J., Alt FW., Denu JM., Jacobson MP., Verdin E. (2010) SIRT3 Deacetylates Mitochondrial 3-Hydroxy-3 Methylglutaryl CoA Synthase 2 and Regulates Ketone Body Production. Cell Metab. 12(6): 654-661.

[37] Warburg O. (1956) On the origin of cancer cells. Science.123:309-314.

[38] Tong X., Zhao F., Thompson CB. (2009) The molecular determinants of de novo nucleotide biosynthesis in cancer cells. Curr Opin Genet Dev.19:32-37.

[39] Gatenby RA., Gillies RJ. (2004) Why do cancers have high aerobic glycolysis? Nat Rev Cancer. 4:891-899.

[40] Kaelin WG Jr., Ratcliffe PJ. (2008) Oxygen sensing by metazoans: the central role of the HIF hydroxylase pathway. Mol Cell. 30:393-402.

[41] Semenza GL. (2010) HIF-1: upstream and downstream of cancer metabolism. Curr Opin Genet Dev. 20:51-56.

[42] Tennant DA., Duran RV., Gottlieb E. (2010) Targeting metabolic transformation for cancer therapy. Nat Rev Cancer.10:267-277.

[43] Gottlieb E., Tomlinson IP. (2005) Mitochondrial tumour suppressors: a genetic and biochemical update. Nat Rev Cancer. 5:857-866.

[44] Zhao S., Lin Y., Xu W., Jiang W., Zha Z., Wang P., Yu W., Li Z., Gong L., Peng Y. (2009) Glioma-derived mutations in IDH1 dominantly inhibits IDH1 catalytic activity and induces HIF-1alpha. Science. 324:261-265.

[45] Finley LWS., Carracedo A., Lee J., Souza A., Egia A., Zhang J., Teruya-Feldstein J., Moreira PI., Cardoso SM., Clish CB., Pandolfi PP., Haigis MC. (2011) SIRT3 opposes reprogramming of cancer cell metabolism through HIF1 $\alpha$ destabilization. Cancer Cell. 19(3): 416-428.

[46] Schumacker PT. (2011) SIRT3 Controls Cancer Metabolic Reprogramming by Regulating ROS and HIF. Cancer Cell. 19(3): 299-300.

[47] Hirschey MD., Shimazu T., Jing E., Grueter CA., Collins AM., Aouizerat B., Stančáková A., Goetzman E., Lam MM., Schwer B., Stevens RD., Muehlbauer MJ., Kakar S., Bass NM., Kuusisto J., Laakso M., Alt FW., Newgard CB., Farese Jr RV., Kahn CR., Verdin E. (2011) SIRT3 Deficiency and Mitochondrial Protein Hyperacetylation Accelerate the Development of the Metabolic Syndrome. Mol Cell. 44(2): 177-190.

[48] Wang GL., Jiang BH., Rue EA., Semenza GL. (1995) Hypoxiainducible factor 1 is a basic-helix-loop-helix-PAS heterodimer regulated by cellular $\mathrm{O} 2$ tension. Proc Natl Acad Sci U S A. 92(12):5510-5514.

[49] Webb J., Coleman M., Pugh C. (2009) Hypoxia, hypoxiainducible factors (HIF), HIF hydroxylases and oxygen sensing. Cell Mol Life Sci. 66:3539-3554.

[50] Semenza GL. (2003) Targeting HIF-1 for cancer therapy. Nat Rev Cancer. 3:721-732.

[51] Bell EL., Emerling BM., SJH Ricoult SJH., Guarente L.( 2011) SirT3 suppresses hypoxia inducible factor $1 \mathrm{a}$ and tumor growth by inhibiting mitochondrial ROS production. Oncogene. 30:2986-2996. 
[52] Mattson MP., Liu D. (2002) Energetics and oxidative stress in synaptic plasticity and neurodegenerative disorders. Neuromolecular Med. 2: 215-231.

[53] Zeng J., Yang GY., Ying W., Kelly M., Hirai K., James TL., Swanson RA., Litt L. (2007) Pyruvate improves recovery after PARP-1-associated energy failure induced by oxidative stress in neonatal rat cerebrocortical slices. J Cereb Blood Flow Metab. 27:304-315.

[54] Liu D., Pitta M., Mattson MP. (2008) Preventing NAD(+) depletion protects neurons against excitotoxicity: bioenergetic effects of mild mitochondrial uncoupling and caloric restriction. Ann N Y Acad Sci. 1147:275-282.

[55] Wang S., Xing Z., Vosler PS., Yin H., Li W., Zhang F., Signore AP., Stetler RA., Gao Y., Chen J. (2008) Cellular NAD replenishment confers marked neuroprotection against ischemic cell death: role of enhanced DNA repair. Stroke. 39:2587-2595.

[56] Yu SW., Andrabi SA., Wang H., Kim NS., Poirier GG., Dawson TM., Dawson VL. (2006) Apoptosisinducing factor mediates poly(ADP-ribose) (PAR) polymer-induced cell death.Proc Natl Acad Sci USA. 103(48):18314-18319.

[57] Liu D., Gharavi R., Pitta M., Gleichmann M., Mattson MP. (2009) Nicotinamide prevents NAD + depletion and protects neurons against excitotoxicity and cerebral ischemia: NAD+ consumption by SIRT1 may endanger energetically compromised neurons. Neuromolecular Med. 11: 28-42.

[58] Ying W., Sevigny MB., Chen Y., Swanson RA. (2001) Poly (ADP-ribose) glycohydrolase mediates oxidative and excitotoxic neuronal death. Proc Natl Acad Sci USA. 98:12227-12232.

[59] Yu SW., Wang H., Poitras MF., Coombs C., Bowers WJ., Federoff HJ., Poirier GG., Dawson TM., Dawson VL. (2002) Mediation of poly (ADP-ribose) polymerase-1-dependent cell death by apoptosis-inducing factor. Science. 297:259-263

[60] Alano CC., Ying W., Swanson RA. (2004) Poly(ADP-ribose) polymerase-1- mediated cell death in astrocytes requires $\mathrm{NAD}+$ depletion and mitochondrial permeability transition. $\mathrm{J}$ Biol Chem. 279:18895-18902.

[61] Alano CC., Tran A., Tao R., Ying W., Karliner JS., Swanson RA. (2007) Differences among cell types in $\mathrm{NAD}(+)$ compartmentalization: a comparison of neurons, astrocytes, and cardiac myocytes. J Neurosci Res. 85:3378-3385.

[62] Kim SH., Lu HF., Alano CC. (2011) Neuronal Sirt3 Protects against Excitotoxic Injury in Mouse Cortical Neuron Culture. PLoS ONE. 6(3): e14731.

[63] Ballatori N., Krance SM., Notenboom S., Shi S., Tieu K., Hammond CL. (2009) Glutathione dysregulation and the etiology and progression of human diseases. Biol Chem. 390:191-214.

[64] Ishigami M., Hiraki K., Umemura K., Ogasawara Y., Ishii K., Kimura H. (2009) A source of hydrogen sulfide and a mechanism of its release in the brain. Antioxid Redox Signal. 11:205-214,

[65] Tang G., Wu L., Wang R. (2010) Interaction of hydrogen sulfide with ion channels. Clin Exp Pharmacol Physiol. 37:753- 763 .

[66] Wallace JL. (2010) Physiological and pathophysiological roles of hydrogen sulfide in the gastrointestinal tract. Antioxid Redox Signal. 12: 1125-1133.

[67] Aggarwal BB., Gehlot P. (2009) Inflammation and cancer: How friendly is the relationship for cancer patients? Curr Opin Pharmacol. 9:351-369.

[68] Lee S., Park Y., Zuidema MY., Hannink M., Zhang C. (2011) Effects of interventions on oxidative stress and inflammation of cardiovascular diseases. World J Cardiol. 3:18-24.

[69] Pan LL., Liu XH., Gong QH., Wu D., Zhu YZ. (2011) Hydrogen sulfide attenuated tumor necrosis factor alphainduced inflammatory signaling and dysfunction in vascular endothelial cells. PLoS One. 6: e19766.

[70] Kida K., Yamada M., Tokuda K., Marutani E., Kakinohana M., Kaneki M., Ichinose F. (2011) Inhaled hydrogen sulfide prevents neurodegeneration and movement disorder in a mouse model of Parkinson's disease. Antioxid Redox Signal. 15:343-352.

[71] Fiorucci S., Distrutti E., Cirino G., Wallace JL. (2006) The emerging roles of hydrogen sulfide in the gastrointestinal tract and liver. Gastroenterology 131: 259-271.

[72] Aminzadeh MA., Vaziri ND. (2012) Downregulation of the renal and hepatic hydrogen sulfide (H2S)-producing enzymes and capacity in chronic kidney disease. Nephrol Dial Transplant. 27:498-504.

[73] Lin CC., Yin MC. (2008) Antiglycative and anti-VEGF effects of S-ethyl cysteine and S-propyl cysteine in kidney of diabetic mice. Mol Nutr Food Res. 52:1358-1364.

[74] Sen U., Basu P., Abe OA., Givvimani S., Tyagi N., Metreveli N., Shah KS., Passmore JC., Tyagi SC. (2009) Hydrogen sulfide ameliorates hyperhomocysteinemia-associated chronic renal failure. Am J Physiol Renal Physiol. 297:F410-419.

[75] Morsy MA., Ibrahim SA., Abdelwahab SA., Zedan MZ., Elbitar HI. (2010) Curative effects of hydrogen sulfide against acetaminophen-induced hepatotoxicity in mice. Life Sci. 87:692-698.

[76] Olson KR. (2009) Is hydrogen sulfide a circulating "gasotransmitter" in vertebrate blood? Biochim Biophys Acta. 1787:856-863.

[77] Nagy P., Winterbourn CC. (2010) Rapid reaction of hydrogen sulfide with the neutrophil oxidant hypochlorous acid to generate polysulfides. Chem Res Toxicol. 23:1541-1543.

[78] Kimura Y., Goto Y., Kimura H. (2010) Hydrogen sulfide increases glutathione production and suppresses oxidative stress in mitochondria. Antioxid Redox Signal. 12: 1-13.

[79] Stasko A., Brezova V., Zalibera M., Biskupic S., Ondrias K. (2009) Electron transfer: A primary step in the reactions of sodium hydrosulphide, an H2S/HS(-) donor. Free Radic Res. 43:581-593.

[80] Olson KR., Whitfield NL. (2010) Hydrogen sulfide and oxygen sensing in the cardiovascular system. Antioxid Redox Signal. 12:1219-1234.

[81] Osborne NN., Ji D., Abdul Majid AS., Fawcett RJ., Sparatore A., Del Soldato P. (2010) ACS67, a hydrogen sulfide-releasing derivative of latanoprost acid, attenuates retinal ischemia and oxidative stress to RGC-5 cells in culture. Invest Ophthalmol Vis Sci. 51: 284-294. 
[82] Bass SE., Sienkiewicz P., Macdonald CJ., Cheng RY., Sparatore A., Del Soldato P., Roberts DD., Moody TW.., Wink DA., Yeh GC. (2009) Novel dithiolethione-modified nonsteroidal anti-inflammatory drugs in human hepatoma HepG2 and colon LS180 cells. Clin Cancer Res. 15:19641972.

[83] Mantovani G., Madeddu C., Maccio A., Gramignano G., Lusso MR., Massa E., Astara G., Serpe R. (2004) Cancerrelated anorexia/cachexia syndrome and oxidative stress: An innovative approach beyond current treatment. Cancer Epidemiol Biomarkers Prev. 13:1651-1659.

[84] Nian H., Delage B., Ho E., Dashwood RH. (2009) Modulation of histone deacetylase activity by dietary isothiocyanates and allyl sulfides: Studies with sulforaphane and garlic organosulfur compounds. Environ Mol Mutagen. 50:213-221.
[85] Droge W., Kinscherf R. (2008) Aberrant insulin receptor signaling and amino acid homeostasis as a major cause of oxidative stress in aging. Antioxid Redox Signal. 10:661-678.

[86] Nimni ME., Han B., Cordoba F. (2007) Are we getting enough sulfur in our diet? Nutr Metab (Lond). 4: 24.

[87] Howard EW., Ling MT., Chua CW., Cheung HW., Wang X., Wong YC. (2007) Garlic-derived S-allylmercaptocysteine is a novel in vivo antimetastatic agent for androgen-independent prostate cancer. Clin Cancer Res. 13:1847-1856.

[88] Ma K., Liu Y., Zhu Q., Liu CH., Duan JL., Tan BK., Zhu YZ. (2011) H2S donor, S-propargyl-cysteine, increases CSE in SGC-7901 and cancer-induced mice: Evidence for a novel anti-cancer effect of endogenous H2S? PLoS One 6:e20525. 